\title{
Article
}

\section{GPs' views and perspectives on patient non-adherence to treatment in primary care prior to suicide}

Pooja, Saini, Chantler, Khatidja and Kapoor, Navneet

Available at http://clok.uclan.ac.uk/17243/

Pooja, Saini, Chantler, Khatidja ORCID: 0000-0001-9129-2560 and Kapoor, Navneet (2017) GPs' views and perspectives on patient non-adherence to treatment in primary care prior to suicide. Journal Of Mental Health . ISSN 0963-8237

It is advisable to refer to the publisher's version if you intend to cite from the work. http://dx.doi.org/10.1080/09638237.2017.1294736

For more information about UCLan's research in this area go to http://www.uclan.ac.uk/researchgroups/ and search for < name of research Group>.

For information about Research generally at UCLan please go to http://www.uclan.ac.uk/research/

All outputs in CLoK are protected by Intellectual Property Rights law, including Copyright law. Copyright, IPR and Moral Rights for the works on this site are retained by the individual authors and/or other copyright owners. Terms and conditions for use of this material are defined in the policies page.

\section{CLoK}

Central Lancashire online Knowledge www.clok.uclan.ac.uk

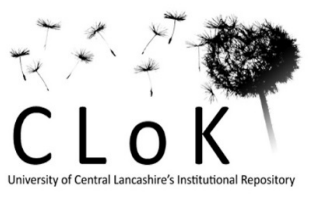


GPs' views and perspectives on patient non-adherence to treatment in primary care prior to suicide

Pooja, Saini ${ }^{1}$

${ }^{1}$ NIHR CLAHRC NWC, Department of Health Services Research, University of Liverpool Khatidja, Chantler ${ }^{2}$

${ }^{2}$ School of Social Work, University of Central Lancashire

Navneet, Kapur ${ }^{3}$

${ }^{3}$ Centre for Mental Health and Risk, University of Manchester

Correspondence: Pooja Saini, NIHR CLAHRC NWC, Department of Health Services Research, $2^{\text {nd }}$ Floor, Waterhouse Building, Brownlow Hill, University of Liverpool, L69 3GS. Tel: 0151794 4860. Email: pooja.saini@liverpool.ac.uk 
Non-adherence to treatment prior to suicide

\begin{abstract}
Background

Individuals who do not adhere to their treatment regimens may be at greater risk of suicide but these issues are relatively unexplored in primary care services.
\end{abstract}

\begin{abstract}
Aim
To explore GPs' views and perspectives on the reasons why patients who were in contact with mental health services in the year prior to suicide were non-adherent to treatment prior to their death.
\end{abstract}

\title{
Method
}

198 semi-structured face-to-face interviews with GPs of people who had died by suicide. Interviews were transcribed verbatim and analysed using a thematic approach.

\section{Results}

The following themes were conceptualised from GP interviews: (i) 'Lack of insight or denial?' relates to what GPs perceived as their patients lack of insight into their psychiatric illness; (ii) 'Lack of treatment choices', discusses GPs' understanding of patient treatment choices; (iii) 'Services for comorbidity and dual diagnosis', refers to treatment availability for suicidal patients with two or more mental health diagnoses.

\section{Conclusion}

For suicide prevention, it is crucial for health professionals to assess patients and manage the possible causes of non-adherence. Policies for increasing treatment adherence, improving services for dual diagnosis or providing alternative treatments to meet individual patient needs may help to achieve the best health outcomes and could potentially prevent suicide.

\section{Keywords}

Suicide, non-adherence, primary care, secondary care, mental health 
Non-adherence to treatment prior to suicide

\section{Introduction}

Primary care is a key setting for suicide prevention as many patients visit their GP in the weeks leading up to their death (DH 2012; NCISHa, 2014; Pearson et al., 2009). Comparatively little is known about GPs' perspectives on treatment adherence and the management of suicidal patients prior to their death (Saini et al., 2015). Non adherence to treatment is recognized by the World Health Organization [WHO], which defines therapeutic adherence as "the extent to which a person's behavior corresponds with agreed recommendations from a health care provider (WHO, 2003). Non-adherence is a key challenge in the management of suicidal patients and in suicide prevention.

Non-adherence to psychotropic medication may be associated with stigma, social isolation, comorbid substance misuse, and the effect of symptom domains on adherence such as positive and negative symptoms, lack of insight, depression, side effects and cognitive impairment (Haddad et al., 2014; Higashi et al., 2013; Novick et al., 2010). Patients with poor pre-morbid cognitive capacity are more likely to stop taking anti-psychotics within the first year of their illness than other patients (Higashi et al., 2013). However, the emphasis placed on the biomedical model alone for the treatment of people with a psychiatric diagnosis with antipsychotic medication has been questioned. Medical labels and side effects can have a negative impact on some individuals and may influence their decision to not adhere to medication or attend appointments with mental health professionals (Bentall 2009; Dowrick $\&$ Frances, 2013). To date, interventions to improve adherence have included psychosocial interventions, psycho-education, antipsychotic long-acting injections, electronic reminders, service-based interventions, and financial incentives. Effectiveness of these interventions has been reported, particularly when tailored to meet an individual patient's need (Haddad et al., 2014; Higashi et al., 2013). 
Non-adherence to treatment prior to suicide

There is limited research on non-adherence to treatment and completed suicide (NCISH 2014b). Most studies have been limited to secondary care settings and in many cases are focussed on patients with schizophrenia or bipolar disorder (Åkerblad et al., 2008; Aldridge 2011; Goff et al., 2010; Haddad et al., 2014; Hawton et al., 2005; Higashi et al., 2013; Hong et al., 2011; Novick et al., 2010; Tacchi \& Scott, 2005). To our knowledge, no studies have investigated non-adherence to treatment prior to suicide within primary care.

The overall aim of this study was to explore GPs' views and perspectives on the reasons why patients who were in contact with mental health services in the year prior to suicide were non-adherent to treatment prior to suicide and strategies for managing such patients in primary care. 
Non-adherence to treatment prior to suicide

\section{Method}

\section{Sample and setting}

The sample for this study included 336 individuals residing in the North West of England who had been in contact with mental health services before they had died by suicide between 1st January 2003 and 30th June 2007. The sample was obtained from The National Confidential Inquiry database (The Inquiry). The named GP for each patient in the sample was assigned a consecutive number ranging between GP1 to GP336.

\section{Collection of primary care and interview data}

All patients' named GP were contacted by letter to participate in the study. GP information was obtained from administrative departments of NHS Trusts of coroner files. One-hundred and ninety-eight GPs agreed to take part in the interview aspect of the study. Interviews took place in the GP surgeries and were recorded with permission and then transcribed verbatim. Where GPs were unavailable, practices were contacted to nominate a suitable alternative GP that had treated the patient in the year prior to death. All interviewees were sent a participant information sheet and indicated their willingness to participate by completing a consent form.

A medical records proforma and interview schedules were used to collect data. These were adapted from tools used in previous research (Pearson et al., 2009) and included details of physical and mental health problems reported in all consultations and treatment offered in the year before death (specifically the final consultation). GPs in primary care rated whether patients were non-adherent to treatment (this included not attending health appointments, not taking prescribed medication or having more medication than the recommended dosage), and where possible, reported the reason for non-adherence. The researcher and GP had access to 
Non-adherence to treatment prior to suicide

the written or electronic medical notes in every interview providing a source of contemporaneous data.

All interviews took place in the GPs' surgeries and were audio-recorded where participating GPs consented to this. Case reviews using the proforma took approximately one hour and interviews varied between 20-40 minutes.

\section{Data analysis}

Thematic analysis was used to analyse GP interview transcripts and was selected as an appropriate method for examining the interview data because it provides a way of getting close to the data and developing a deeper appreciation of the content (Braun \& Clarke, 2006). Data was written on the proformas designed for the interview data, under each relevant section. All data was checked for errors by listening back to the audio-recording and reading the proformas simultaneously. PS conducted majority of the interviews (81\%) and listened back to the audio-recorded interviews to become familiar with the whole data set. This familiarisation process was essential in cases where PS was not the researcher present during the interview. Familiarisation through reading and making notes in this way enabled PS to find her way easily around hundreds of pages of data later in the analysis.

The main themes and codes of interest were determined by using the steps recommended by Braun and Clarke (2006): listening to interview recordings and reading each transcript several times to establish familiarity with the whole interview and generating descriptive codes to represent the main themes. Initially PS coded the proformas completed with the interview data and underlined interesting segments of text - this could range from only a few words, to parts of sentences or whole paragraphs. PS recorded more detailed notes and ideas, for 
Non-adherence to treatment prior to suicide

example questions to bear in mind as the analysis proceeded, and ideas for explanations or patterns in the data. PS emphasised interesting parts of the data that they felt were worth coding or noting. In this approach, one piece of data (e.g. one statement, one theme) was taken and compared with all information for similarities or differences. This ongoing analysis refined the specifics and formulated the conceptual name of each of the three themes. The final part of the analysis was the selection of the interview extracts, relating the analysis to the research question and literature. The process of refinement and validation of findings was conducted through a collaborative exercise creating iterative feedback loops. Transcripts were examined for one group of the data set where patients were reported to be non-adherent to treatment by GPs. The data were interpreted and reanalysed within the thematic framework to interpret and structure the component statements. The analysis was principally conducted by the primary researcher (PS) and emergent themes and key issues were discussed with the secondary researcher $(\mathrm{KC})$. 
Non-adherence to treatment prior to suicide

\section{Results}

\section{Characteristics of the sample}

Between January 2003 and June 2007, 336 patient suicides were recorded in the North West of England; approximately $6 \%$ of the entire national sample of patient suicides $(n=5,552)$. Of the 336 GPs who were invited to participate in the study, 198 (59\%) consented to be interviewed and 135 did not participate due to being retired, lack of time, having left the practice or they had died and two GPs changed their minds about participating at the time of the interviews. Where the patient's own GP was unavailable, practices were contacted to nominate a suitable alternative GP that had treated the patient in the year prior to death. This occurred for 54 of the 198 patient cases included in this study (27\%); however for 15 out of the 54 patient cases, the nominated GP was the last GP the patient had seen (27\%). Semistructured face-to-face interviews were conducted with GPs between January 2005 and October 2009. Of the GPs, 54 were female and 144 were male. The length of time since qualifying ranged from 8 to 40 years, with an average of 23 years.

Of the 198 GP interviews, data were obtained for 96 (48\%) patients who were non-adherent to treatment. GPs reported that $95(99 \%)$ patients received additional specialist mental health care in the year prior to their death. GPs were involved in urgent referrals or emergency admissions for $15(16 \%)$ patients at their final consultation and GPs encouraged patients to adhere to medication or attend mental health appointments in $15(16 \%)$ cases. The most common reason for patient non-adherence to treatment was comorbidity (see Graph 1). Other reasons for non-adherence were: side effects, stigma of taking medication; patients disbelieving they had a psychiatric illness; the belief that the medication was not working; feeling better; having more than the prescribed dosage; encouragement by others not to take 
Non-adherence to treatment prior to suicide

"mind-controlling" substances; wanting to get pregnant; not being able to drive or simply running out of medication.

Following the thematic analysis process, three inter-related themes were conceptualised that illustrate the reasons GPs reported for non-adherence to treatment prior to patient suicide. The first theme related to what GPs perceived as their patients lack of insight into their psychiatric illness and was conceptualised as 'Lack of insight or denial?'. The second theme 'Lack of treatment choices' related to GPs' understanding of patient treatment choices with regards to medication, which include living with the side effects of medication or living without any treatment due to the lack of alternative treatment options. The third theme identified was 'Services for comorbidity and dual diagnosis' and related to treatment availability (or lack of availability) for suicidal patients with two or more diagnoses. Each of these themes is developed below.

\section{Lack of insight or denial?}

There is growing evidence that a lack of insight may be an important reason why individuals with severe psychiatric disorders do not take medication for their illness (Rickleman 2004; Schwartz 1998). This GP's comment is representative of GPs perspectives that some patients lack insight into their condition:

"She was not compliant with medication and had poor insight in her last episode before her death...the community mental health team were involved in following her up and trying to make sure she was taking her medication but she just deteriorated..." (GP50). Evaluating insight is crucial for GPs who may be making a psychiatric diagnosis and for assessing potential adherence to treatment. A patient's lack of insight or denial of psychiatric symptoms is one of the more troubling aspects of treatment as it can prevent a person from getting the help they need. Previous studies suggest that one method of handling this is to rely 
Non-adherence to treatment prior to suicide

on a "doctor knows best" approach and simply to medicate or hospitalise patients without consent (Davies et al., 2010). However, another approach recommended by Chao and Kawasaki (2007; citied in Amador 2010) relies on listening to the patient, empathising with the patient, agreeing with the patient and partnering with the patient (this approach is signified as LEAP). This method ensures that GP's use the patient's own framework to build rapport and communicate with them.

In this study, GPs reported that $8(7 \%)$ patients (who had at least one psychiatric diagnosis) were convinced they did not need psychotropic medication for their illness or that they had other major physical illnesses (e.g. cancer, aids), although clinical tests proved otherwise;

"Patient was convinced they had a physical health problem and would not believe it was his mental health and so did not comply with medication” (GP77).

Although GPs listened to patient worries about their illness and dealt with them in a thorough manner, patients who usually had one or more psychiatric diagnoses were often difficult to treat. Interestingly, $10(63 \%)$ of the patients who were reported as displaying a lack of insight into their illness had a history of suicidal ideation and/or a history of one or more severe suicide attempt(s).

\section{Lack of treatment choices}

For some patients, several months or years of treatment of psychotherapy or medication are necessary and follow up of these patients is crucial. The recommended treatment duration for the first episode of depression is six to twelve months, but almost half of patients stop taking their antidepressant within three months for various reasons (Grenard et al., 2011; NICE 2004). Metabolic side effects of psychotropic medications, particularly for schizophrenia and bipolar disorder, may contribute to heart disease and strokes and this along with more 
Non-adherence to treatment prior to suicide

immediate effects such as weight gain may affect some patients' decision for not wanting to take long-term medications (Hert et al., 2011). However, it seemed that with some patients, medication or inpatient treatment was unhelpful psychologically and encouraging patients was clearly not the right strategy as this was not going to eradicate the medication's side effects. In this study, GPs reported that $30 \%$ of patients stopped taking medications due to side effects that sometimes had a major impact on quality of life such as weight gain or feeling sluggish. GPs spoke of their patients' worry about not being able to drive, having to stop drinking alcohol, reduction in fertility and/or sex drive;

"She did not take medication because she wanted to get pregnant and she thought she couldn't whilst she was on that medication" (GP101).

Patients' were encouraged to take their prescribed medication by GPs when they discontinued or disclosed discontinuing and on many occasions GPs reported that patients were discharged from psychiatric services too soon - sometimes GPs were not informed, involved in or aware of their patient's discharge plan.

Enhancing medication adherence (or preventing non-adherence) is an important treatment goal for GPs. GP recognition and prevention of factors that could lead to patient nonadherence is vital, as well as having alternative treatment options to medication for some patients. However, many GPs reported the difficulties they faced to provide alternative options for patients who communicated that a treatment was not working for them:

"Should have been possible in this case to put [the]deceased in hospital or a therapeutic community as she requested but services in this area are poor particularly for those who need counselling...Needed counselling but psychology referral for this patient was refused. Her medication was just helping her get by" (GP210). 
Non-adherence to treatment prior to suicide

GPs reported their frustration of not being able to meet individual patient's needs when they were requesting treatments that should be available and that the GP felt were necessary. GPs suggested that offering evidence based psychosocial interventions was more appropriate for some patients, particularly those who had been abused or were perpetrators of abuse (a proportion of patients in this study were abused as children and/or were perpetrators of abuse). However, GPs reported a lack of access to alternative treatment options, such as psychotherapy, thus limiting what GPs could offer and this impacted on patient choice.

\section{Services for comorbidity and dual diagnosis}

GPs reported the management of non-adherence in primary care was complicated by the many health and social issues that this group of patients had. This influenced their treatment behaviour and many patients were reported to have chaotic lifestyles. GPs highlighted that some patients used alcohol and/or drugs as a way of self-medicating, had done so for many years, and emphasised that patients required different services to meet their individual health needs prior to suicide.

GPs spoke of patients with chaotic lifestyles who had ongoing input from primary and secondary mental health care services but who still went on to die by suicide. For many of these cases GPs felt that the patient's lifestyle affected their ability to obtain the treatment they needed;

"He had a chaotic lifestyle and had taken drugs for years. He did not attend his appointments for his depots although the nurse did try her best to see him" (GP67).

These patients were more likely to be from socially deprived areas, misused substances, had a history of self-harm and/or suicide attempts and have a history of physical or sexual abuse. GPs faced tensions when managing patients who have multiple health and social issues, 
Non-adherence to treatment prior to suicide

particularly when they reported being unsupported by specialist mental health services. In this study, many patients may have benefited from years of psychotherapy alongside psychotropic medication and/or rehabilitation programs for substance misuse/dependency. However, there appear to be obstacles and barriers in accessing services across the care pathway from when a patient consults with a GP to receiving specialist mental health care. Surprisingly, these problems still existed when GPs referred patients displaying risk factors of suicide to specialist mental health services.

Dual diagnosis was reported for $69 \%$ of patients who were non-adherent to treatment and $80 \%$ of patients had two or more mental health diagnoses (see graph 2). Service availability was an issue in most areas and GPs recognised that dual diagnosis was often a cause for nonadherence to treatment, particularly for attending or being available for mental health appointments;

"He could not attend appointments and received care as an inpatient, from outpatients and the community alcohol team but he did not want to engage with alcohol services... all his appointments were related to depression and alcoholism, followed by selfharm. He mostly phoned for consultations and also refused to see me when he was drunk." (GP251)

In this sample of patients, GPs conducted numerous home visits as some patients could not leave their home due to being agoraphobic or intoxicated; however they reported being let down when these patients were not assessed or followed up by mental health services due to not attending or 'opting in' for appointments. GPs perceived that it was sometimes a vicious circle as some patients were refused admission because they were intoxicated and were left for primary care to manage alone or patients were left to fend for themselves; 
Non-adherence to treatment prior to suicide

“...due to alcohol issues, psychiatry would not admit him. It was difficult to get him to come to appointments but family took him and GP did home visits" (GP239).

Nearly half of the GPs (48\%) reported needing quicker and better services for comorbid and dual diagnosis (44\%) and highlighted the need for services to 'strike while the iron's hot' particularly when patients, usually men, consulted for treatment for drug or alcohol dependency in primary care;

He was in and out of prison and while in prison he had two suicide attempts. He always struggled with alcohol and depression was secondary to this. He would come in [to primary consultations with GP] and want help NOW and then DNA if treatment took time. He had an extremely chaotic use of the system." (GP223).

GPs believed that many missed appointments that resulted in patients being removed from mental health services were alcohol related and that community psychiatric nurses should be brought back into GP surgeries as patients could be seen immediately for an assessment and referred for treatment within an appropriate service sooner. 
Non-adherence to treatment prior to suicide

\section{Discussion}

\section{Main findings}

GPs reported the dilemma they faced when managing patients who: (i) were convinced that they did not have a mental health diagnosis; (ii) found side effects of psychotropic medications intolerable; (iii) had a dual diagnosis or comorbidity; and, (iv) had a lack of access to services or lack of support from mental health professionals. GPs reported that some patient suicides may have been prevented had these services been available. GPs reported that nearly a fifth of the patients who were diagnosed with agoraphobia or were socially isolated, were often more vulnerable individuals requiring additional support. However many of these patients were not being followed-up as a result of being unable to attend appointments and were often left alone to fend for themselves or solely under the management of primary care. These issues were heightened in patients with multiple mental health diagnoses and substance misuse issues. Another challenge faced by GPs was for patients who appeared to accept treatment offered in primary care but who were actually non adherent to the recommended treatment. In these consultations, patients may be behaving as they feel they are expected to behave in front of the GP (thus accepting the treatment offered) but what the patient did following the consultation was out of the GPs control; thus GPs would be unaware of the need to intervene. This issue further highlights the need of ongoing monitoring and assessment of patients who are vulnerable and at risk of suicide as a joint responsibility between primary and secondary care.

\section{Methodological considerations}

The findings in this study should be interpreted in the context of a number of methodological limitations. This is essentially a study of the primary care management of a secondary care sample, thus the issues identified may not apply to all people who die by suicide in primary 
Non-adherence to treatment prior to suicide

care. The adherence data may be under reported as primary care or secondary care services may not have known whether people were adherent to treatment or not (an ascertainment bias). Some of the data were retrospectively collected so there is also the possibility of recall bias, although this is unlikely to have a significant impact upon the medical records data. Previous studies have identified variations in accuracy or the amount of detail provided in case records (Mistry et al., 2005; Jordan et al., 2004); however, good agreement has also been found when comparing GP records and patient self-report questionnaires for the mean number of consultations in both sources (Mistry et al., 2005). In addition, our primary care data was supplemented by interviews with GPs and our secondary care data collection (by means of a dedicated proforma) was collected directly from the clinicians caring for the patients.

Our findings may not be representative of the rest of the UK although many of the issues we identified are likely to apply across services. It should also be noted that our data are now several years old. As a consequence some of the study findings might not necessarily reflect current clinical practice.

\section{Clinical and Research implications}

Whilst many of the issues reported in this study are not specific to suicide; they may have specific meanings in relation to suicide prevention (Hawton et al., 2005; Goff et al., 2010; Tacchi and Scott, 2005; Novick et al., 2010; Higashi et al., 2013; Hong et al., 2011; Apantaku-Olajide et al., 2011). Strengthening adherence may include more consistent training for GPs in understanding the reasons for patient non-adherence to treatment in order to provide the most appropriate treatment. GPs may benefit from further training in encouraging patients to discuss their symptoms and side effects with mental health 
Non-adherence to treatment prior to suicide

professionals, rather than simply to stop taking medication as this may prevent serious adverse events such as suicide attempts, relapse, treatment drop-out and hospitalisation. However, improving medication adherence is challenging (Aldridge 2011) because alternative treatment options may be unable to meet each patient's individual needs. Improving communication between primary and secondary care for the ongoing risk management of patients who are non-adherent to treatment and who might be at risk of suicide and is therefore critical (Saini et al, 2014).

Service user literature on psychotropic medication suggests it can be frightening for some individuals and can feed into their mental illness and ultimately be more unhelpful psychologically (Gault et al., 2013). Further, additional support for patients may not necessarily improve adherence as the side effects will remain intolerable. Therefore, to establish the underlying reasons for patients' worry and fear about their health, GPs may have benefited from referring patients for psychotherapy. Perhaps, a shift in GP attitudes from a perception that patients lack insight into their mental illness to recognition that there may be more suitable treatment options that meet an individual's needs could be more useful for suicide prevention as power relations associated with medical discourses are apparent in these accounts with the use of labels such as 'lacking insight'.

Our findings were consistent with previous studies with respect to the prevalence of alcohol and drug dependency, distressing side effects, chaotic lifestyles, comorbid disorders and dual diagnosis in patients who were non-adherent to treatment (Hawton et al., 2005; Hunt et al., 2015); Goff et al., 2010; Tacchi and Scott, 2005; Novick et al., 2010; Higashi et al., 2013; Gold 2009). Patients with dual diagnosis can be more difficult to engage within services and this can be due to not wanting to take medication alongside drug or alcohol use (Higashi et 
Non-adherence to treatment prior to suicide

al., 2013). This was also confirmed in this study. Strengthening services for comorbidity and dual diagnosis may improve the engagement of patients, particularly young patients, with primary and secondary care and could contribute to suicide prevention (Magura 2008; Magura et al., 2008)

These findings indicate that integrated services involving better communication and collaborative care between primary care, substance misuse and mental health services may benefit patients at risk of suicide. Research suggests that broad spectrum diagnosis and concurrent therapy will lead to more positive outcomes for patients with comorbid conditions. Steady progress is being made on new and existing treatment options for comorbidity in a variety of settings, however access to these services is limited (Saini et al., 2010). This area warrants further research to investigate and understand the engagement and treatment differences in patients who are non-adherent to treatment to improve practice.

\section{Conclusion}

Non-adherence to treatment regimens may be difficult to manage in primary care. For suicide prevention, it is crucial for health professionals to assess patients and manage the possible causes of non-adherence. Possible strategies to improve treatment adherence in order to contribute to suicide prevention could include: 1) GP training in the assessment of patients who are non-adherent and improved management of the possible causes of non-adherence; 2) increasing availability of alternative treatment options to medication in order to improve patient choice in primary care; and, 3) providing specialty services to assist GPs in treating patients with multiple mental health diagnoses, including drug and alcohol dependency. Follow-up visits are also very important for enhancing and monitoring adherence and should include assessing a patient response to treatment, evaluating possible side effects and 
Non-adherence to treatment prior to suicide

management by GPs and/or mental health professionals. Clear guidelines need to be in place to deal with patients who do not attend appointments with mental health professionals.

Discharging patients who do not attend appointments should be avoided and joint working should be increased across primary and secondary care within the health service. 
Non-adherence to treatment prior to suicide

\section{Acknowledgements}

We thank all participating GPs and the PCTs that allowed us access to medical records and participated in interviews. The study was carried out as part of the National Confidential Inquiry into Suicide and Homicide by People with Mental Illness. We thank the other members of the research team: David While, Kirsten Windfuhr, Alyson Williams, Anna Pearson, Damien Da Cruz, Caroline Miles, Harriet Bickley, Jimmy Burns, Isabelle Hunt, Rebecca Lowe, Phil Stones, Pauline Turnbull, Sandra Flynn, Cathy Rodway, Alison Roscoe and Kelly Hadfield. We acknowledge the help of Professor Louis Appleby, Professor Jenny Shaw, health authorities, trust contacts and consultant psychiatrists for completing the questionnaires.

\section{Declaration of interests}

NK was Chair of the Guideline Development Group for the National Institute for Clinical Excellence (NICE) guidelines for the longer term management of self-harm, currently chairs the depression in adults' guideline, and sits on the Department of Health's National Suicide Prevention Strategy Advisory Group.

This study was initiated while NCISH was funded by the National Patient Safety Agency and funding was transferred to the Healthcare Quality Improvement Partnership in 2011.

\section{Competing Interests}

The views expressed are those of the author(s) and not necessarily those of the NHS, the NIHR or the Department of Health.

\section{Ethics}

Ethical approval was granted by the North-west Research Ethics Committee (REC reference: $02 / 8 / 74$ ) for the Inquiry as a whole. Individual $R \& D$ approvals were also obtained from all the relevant Mental Health Trusts included in the study. 
Non-adherence to treatment prior to suicide

\section{References}

Åkerblad, A.C., Bengtsson, F., Holgersson, M., von Knorring, L., Ekselius, L. (2008) Identification of primary care patients at risk of non-adherence to antidepressant treatment. Patient preference and adherence, 2, 379.

Aldridge, M.A. (2011) Addressing non-adherence to antipsychotic medication: a harm reduction approach. Journal of Psychiatric and Mental Health Nursing, 1-12.

Amador, X. (2010) I Am Not Sick. I Don't Need Help. New York, Vida.

Bentall, R.P. (2009) Doctoring the mind. Why psychiatric treatments fail. London, Allen Lane.

Braun, V., Clarke, V. (2006) Using thematic analysis in psychology. Qualitative Research in Psychology, 3, 77-101.

Davies, P., Taylor, F., Beswick, A., Wise, F., Moxham, T., Rees, K., Ebrahim, S. (2010) Promoting patient uptake and adherence in cardiac rehabilitation. Cochrane Database of Systematic Reviews; issue 7; art no: CD007131.

Department of Health. (2012) Preventing suicide in England: a cross-government outcomes strategy to save live, https://www.gov.uk/government/uploads/system/uploads/attachment_data/file/216928/Pr eventing-Suicide-in-England-A-cross-government-outcomes-strategy-to-save-lives.pdf (assessed on 16/12/15).

Dowrick, C., Frances, A. (2013) Medicalising unhappiness: new classification of depression risks more patients being put on drug treatment from which they will not benefit. BMJ, 347, f7140.Fawcett J. (1995) Compliance: Definitions and key issues. Journal of Clinical Psychiatry, 56 (suppl 1), 4-8. 
Non-adherence to treatment prior to suicide

Gault, I., Gallagher, A., Chambers, M. (2013) Perspectives on medicine adherence in service users and carers with experience of legally sanctioned detention and medication: a qualitative study. Patient Prefer Adherence, 7, 787-99.

Goff, D.C., Hill, M., Freudenreich, O. (2010) Strategies for improving treatment adherence in schizophrenia and schizoaffective disorder. Journal of Clinical Psychiatry, 71, 20-6.

Gold, L. (2009). Reductions in psychiatry. Canadian Journal of Psychiatry, 54, 506512.

Grenard, J.L., Munjas, B.A., Adams, J.L., Suttorp, M., Maglione, M., McGlynn, E.A. Gellad, W.F. (2011) Depression and medication adherence in the treatment of chronic diseases in the United States: A meta-analysis. Journal of General Internal Medicine, 26, $1175-1182$.

Haddad, P.M., Brain, C., Scott, J. (2014). Nonadherence with antipsychotic medication in schizophrenia: challenges and management strategies. Patient Related Outcome Measures, 5, 43-62. http://doi.org/10.2147/PROM.S42735

Hawton, K., Sutton, L., Haw, C., Sinclair, J., Deeks, J.J. (2005) Schizophrenia and suicide: systematic review of risk factors. The British Journal of Psychiatry, 187, 9-20.

Hert, M.D., Correll, C., Bobes, J., Cetkovich-Bakmas, M., Cohen, D., Asai, I., Detraux, J., Gautam, S., Möller, H.J., Ndetei, D.M., Newcomer, J.W., Uwakwe, R., Leucht, S. (2011) Physical illness in patients with severe mental disorders. Prevalence, impact of medications and disparities in health care. World Psychiatry, 10, 52-77.

Higashi, K., Medic, G., Diez, T. (2013) Medication adherence in schizophrenia: Factors influencing adherence and consequences of non-adherence - a systematic literature review. Therapeutic Advances in Psychopharmacology, 3 (4), 200-218. 
Non-adherence to treatment prior to suicide

Hong, J., Reed, C., Novick, D., Haro, J.M., Aguado, J. (2011) Clinical and economic consequences of medication non-adherence in the treatment of patients with a manic/mixed episode of bipolar disorder: results from the European Mania in Bipolar Longitudinal Evaluation of Medication (EMBLEM) study. Psychiatry Research, 190, (1), $110-4$.

Hunt, I.M., Clements, C., Saini, P., Rahman, M.S., Shaw, J., Appleby, L., Kapur, N., Windfuhr, K. (2016). Suicide after absconding from inpatient care in England: an exploration of mental health professionals' experiences. Journal of Mental Health, DOI: 10.3109/09638237.2015.1124394.

Jordan, K., Porcheret, M., Croft, P. (2004) Quality of morbidity coding in general practice computerised medical records: a systematic review. Family Practice, 21 (4), 396412.

Magura, S. (2008) Effectiveness of dual focus mutual aid for co-occurring substance use and mental health disorders: A review and synthesis of the "Double Trouble" in Recovery evaluation. Substance Use \& Misuse, 43, 1904-1926.

Magura, S., Rosenblum, A., Villano, C.L., Vogel, H.S., Fong, C., Betzler, T. (2008) Dual-focus mutual aid for co-occurring disorders: A quasi-experimental outcome evaluation study. American Journal of Drug and Alcohol Abuse, 34, 61-74.

Mistry, H., Buxton, M., Longworth, L., Chatwin, J., Peveler, R. (2005) Comparison of general practitioner records and patient self-report questionnaires for estimation of costs. European Journal of Health Economics, 50, 261-266.

National Confidential Inquiry into Suicide and Homicide by People with Mental Illness (NCISHa) (2014) Suicide in Primary Care in England: 2002-2011. University of Manchester, Manchester. 
Non-adherence to treatment prior to suicide

National Confidential Inquiry into Suicide and Homicide by People with Mental Illness (NCISHb). Annual Report 2014.

http://www.bbmh.manchester.ac.uk/cmhr/centreforsuicideprevention/nci/reports/Annualr eport2014.pdf (accessed on 16/12/15)

National Institute for Health and Clinical Excellence (2004) Depression: Management of Depression in Primary and Secondary Care - NICE Guidelines CG23. NHS, UK.

Novick, D., Haro, J.M., Suarez, D., Perez, V., Dittmann, R.W. \& Haddad, P.M. (2010) Predictors and clinical consequences of non-adherence with antipsychotic medication in the outpatient treatment of schizophrenia. Psychiatry Research, 176, 109113.

Pearson, A., Saini, P., Da Cruz, D., Miles, C., While, D., Swinson, N., Williams, A., Shaw, J., Appleby, L., Kapur, N.(2009) Primary care contact prior to suicide in individuals with mental illness. British Journal of General Practice, 59 (568), 826-832.

Rickleman, B.L. (2004) Anasognosia in individuals with schizophrenia: Toward recovery of insight. Issues in Mental Health Nursing, 25, 227-242.

Saini, P., Windfuhr, K., Pearson, A., Da Cruz, D., Miles, C., Cordingley, L., While, D., Swinson, N., Williams, A., Shaw, J., Appleby, L., Kapur, N. (2010) Suicide prevention in primary care: General practitioners' views on service availability. BMC Research Notes, 3, 246.22.

Saini, P., While, D., Windfuhr, K., Chantler, K., Kapur, N. (2014). Assessment and management of suicide risk in primary care. Crisis, 18, 1-11.

Saini, P., Chantler, K., Kapur, N. (2015). GPs' perspectives on primary care consultations for suicidal patients. Journal of Health and Social Care in the Community. 24(3):260-9. doi: 10.1111/hsc. 12198 . 
Non-adherence to treatment prior to suicide

Schulberg, H.C., Hyg, M.S., Bruce, M.L., Williams, J.W. Jr, Dietrich, A.J. (2004) Preventing suicide in primary care patients: the primary care physician's role. General Hospital Psychiatry, 26, 337-345.

Schwartz, R.C. (1998) Insight and illness in chronic schizophrenia. Comprehensive Psychiatry, 39 (5), 249-254.

Tacchi, M.J., Scott, J. (2005) Improving adherence in schizophrenia and bipolar disorders. John Wiley and Sons Ltd, Hoboken, N.J.

WHO. Adherence to long-term therapies: evidence for action. Geneva, Switzerland: World Health Organization; 2003. (accessed on 16/12/2015). Available from: http://whqlibdoc.who.int/publications/2003/9241545992.pdf . 